\title{
On the Design of Integrated Air/Ground Automation
}

\author{
Thomas Prevot \\ San Jose State University \\ NASA Ames Research Center \\ Moffett Field, CA 94035, USA \\ tprevot@mail.arc.nasa.gov
}

\begin{abstract}
This paper addresses the design of automation for the primary operators in the air transportation system: air traffic controllers and flight crews. The modernization of the airspace system requires the implementation of extensive integrated automation support in air traffic control facilities and on flight decks. Research in the human factors division at NASA Ames Research Center over the past seven years has focused on prototyping and evaluating both air and ground aspects of envisioned future air traffic management concepts. This paper presents an example of automating an air traffic control task. It expresses lessons learned and views on the process of designing automation for the very complex and distributed air traffic system.
\end{abstract}

Keywords: Human automation interaction, air traffic management, air ground integration.

\section{Introduction}

In December 2004 the Joint Planning and Development Office (JPDO) transmitted the "Integrated National Plan for the Next Generation Air Transportation System" [1] to the United States Congress. The plan stresses the need for a new technology enabled approach to air transportation. It outlines a high-level vision for 2025 that combines increased automation with new procedures to achieve economical, capacity, safety, environmental, and security benefits.

The plan presents a number of operational concept elements that are aimed at tripling sector and airport capacity by 2025 . New avionics will enable aircraft to operate with increasing levels of aircraft autonomy and increase flight deck situational awareness. Automation will make new air traffic management (ATM) concepts possible including shared or distributed separation management. ATM operations are envisioned to rely on end-to-end strategic traffic flow management, data link communication and information sharing to contract quiet and fuel efficient flight profiles between ground automation and airborne flight management systems and minimize adverse weather effects.
Similar objectives are driving forces behind the development of the European ATM Master Plan aimed at creating a coherent and manageable research and implementation path to mobilize and consolidate scarce resources, avoiding unproductive duplication and overlap [2].

These plans call for a rapid modernization of the airspace system and particularly stress the importance of synchronization and harmonization of airborne and ground deployments. Research and development are called upon to pick up the pace and investigate bold changes to the air transportation system quickly, while resources are very limited. Therefore a compromise between in-depth investigation and pragmatic approaches has to be found that enables the progression of air transportation in a safe and secure environment. This paper addresses the unique airspace system problem and current automation trends. It also presents an approach to rapid prototyping and evaluation of future air traffic concepts used in the human factors division at NASA Ames Research Center. Additionally, it discusses lessons learned with regard to the process in general and some specific examples from research on air/ground integration and Distributed Air Ground Traffic Management (DAG-TM) [3].

\section{Human-centered automation or automation-centered humans?}

The current air traffic system relies on air traffic controllers separating a certain number of flights (typically 10 to 18 ) in a predefined airspace sector with very limited automation support. Flight crews are responsible for the safe conduct of their flight and manage their airplane according to the controllers' instructions and clearances using on-board automation. In support of their primary tasks controllers have an airspace-centric traffic view whereas flight crews have an own-ship centric flight path view.

Frequently, excessive air traffic controller workload is considered the primary limiting factor in substantially increasing airspace capacity, i.e. adding more aircraft to a given sector. Consequently, most approaches aimed at 
increasing capacity try to offload tasks from the air traffic controller to other entities. Two main candidates for taking on some or all of these responsibilities are the flight deck and the ground automation. The concept of making the flight crew responsible for separating their aircraft from other traffic is referred to as airborne self-separation [4]. The idea of making the ground automation responsible for separation underlies the Advanced Airspace Concept [5].

It could appear that airborne self-separation implies training pilots as air traffic controllers to take on the new separation task, but this is not what is envisioned by the concept. In contrast, all airborne self-separation concepts require airborne automation to detect traffic conflicts and alert the flight crew, and to provide automated assistance in resolving the conflicts. De facto, both ideas - airborne selfseparation and making the ground automation responsible for separation - assign the primary air traffic control task of separation management to the automation.

Problems for pilots in interacting with engineerdesigned airborne automation have led to developing the principles of "human-centered" automation [6]. In order to avoid repeating the problems of the past it would seem natural to design advanced ground-based systems and new airborne technologies around the principles of humancentered automation. Following these principles we were able to design human-centered decision support tools for pilots and controllers that indicated a great potential for improving air traffic system effectiveness. An example will be given later in the paper.

Unfortunately, tendencies expressed in the current definition of several future plans call for using automationcentered approaches and ultimately replacing the resourcelimited humans with advanced automation. Obviously, human vs. automation resources, limitations and capabilities have been subject to a wide body of research and we don't claim to have any new breakthrough insights into the general subject matter. Nevertheless, the air transportation system is on the verge of undergoing major changes and it appears imperative to make sure -early in the process- that these changes are feasible, operationally viable, safe, and acceptable to all operators. Some of the challenges in automating typical air traffic control tasks will be illustrated in the following section.

\section{Introducing new automation: The delay vectoring example}

A typical problem in today's air traffic environment is delay absorption. When more aircraft arrive at an airport than can be landed immediately, demand exceeds capacity and some aircraft have to be delayed. Since not all aircraft can be delayed within the terminal area surrounding the runways most of the delay is passed back to be absorbed within the air traffic control center outside the terminal area. Metering fixes are used to meter aircraft into the terminal area. Without additional automation controllers can deliver aircraft approximately at a predefined miles-intrail distance from each other. Controllers are trained and experienced with issuing instructions to aircraft to provide these flows. The procedure of providing miles-in-trail feeds works reasonably well as long as traffic streams from different meter fixes do not have to be coordinated.

To improve traffic planning and coordination air traffic facilities in the U.S. and Europe started using automation. Automation like the Center TRACON Automation System (CTAS) [7] with its Traffic Management Advisor (TMA) tool is designed to predict traffic demand and capacity and assign scheduled times of arrival (STA) to arriving aircraft. Instead of delivering aircraft with a certain number of miles-in-trail between each other, controllers are now tasked to deliver aircraft at their STA. Controllers have different options to delay aircraft that they typically use depending on delay magnitude. For delays of more than six minutes aircraft are usually instructed to enter a holding pattern. Delays of one or two minutes are usually handled with speed and altitude changes. The middle range of two to six minutes usually requires lengthening the route of the aircraft. This procedure is often referred to as delay vectoring and will serve as the example for the subsequent discussion.

\subsection{Current day delay vectoring}

In today's environment delaying an aircraft for a few minutes typically involves the following sequence of events.

(1) The air traffic controller detects that the aircraft will arrive earlier than it should using the metering information provided on the display.

(2) The air traffic controller determines an appropriate heading for the aircraft that will lengthen its flight path

(3) The controller instructs the flight crew verbally: "AAA123 turn left heading 120 for metering delay"

(4) The flight crew selects the new heading

(5) The controller monitors the position of the aircraft and the estimated remaining metering delay to call the turn back to the metering fix

(6) The controller instructs the flight crew verbally: "AAA123 proceed direct Waypoint XYZ"

(7) The flight crew uses the "direct to" function in the flight management system (FMS) and re-engages the automation to fly direct to that waypoint 


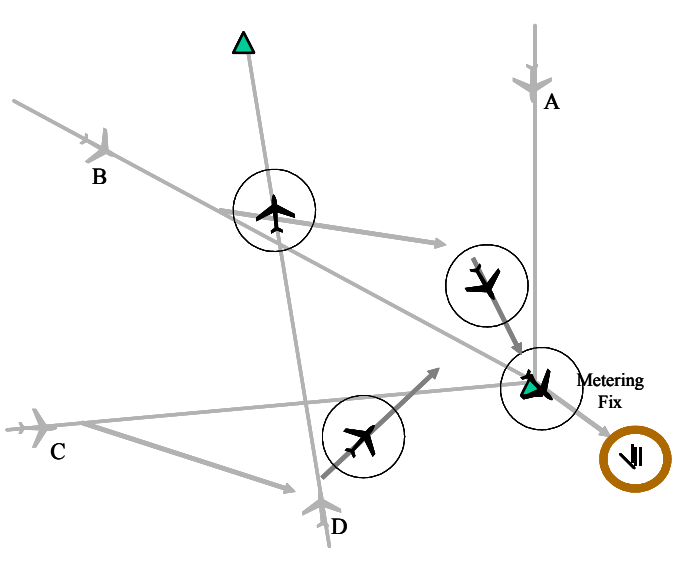

Figure 1. Typical current day arrival problem. Aircraft B and $\mathrm{C}$ have to change their routing to arrive behind aircraft

A. Aircraft D represents an additional traffic constraint

The air/ground procedure is simple and straightforward; all clearances can be easily and unambiguously communicated between the controller and the pilot. The controller does not have to input anything into his or her workstation and the necessary pilot inputs are easy and routine.

The main workload is with the controller, who needs to monitor the delay countdown to call the aircraft's turn back. Therefore, this type of radar vectoring is typically considered imprecise and the resulting flight paths inefficient. Depending on workload and skill the controller may miss the turn-back point and impose unnecessary delay to the aircraft or cause a conflict situation which requires additional workload to be resolved. Another disadvantage of this current day approach is that only the controller has a mental estimate of the aircraft's flight path. The flight crews don't know when the turn back will occur and the ground system as well as the airborne system has no knowledge of the actual flight intent. This causes major difficulties in using any automated functions during this phase like ground based conflict probing or airborne flight path management.

This problem is therefore a prime candidate for introducing decision support automation to aid the controller. Aiding controllers in delay vectoring has been subject to research efforts for more than a decade and there is still no automation in place. Different ground-based approaches will be analyzed in the following sections highlighting some of the difficulties and strengths.

\subsection{Ground-based manual trial planner}

One approach to reduce controller workload and maintain flight path predictability is to use a ground based route planning tool that allows controllers to pre-construct a conflict free delay path graphically [8]. During the path construction process the controller receives feedback about the delay that the new path would absorb and potential conflicts along the way. Once the controller accepts the path he or she would then send it as flight plan amendment to the ground based automation. Therefore, better flight path predictability can be achieved if the aircraft can follow the trial planned flight path precisely. However, after several field tests and simulations in 1999 McNally et al. [8] concluded: "It was found during simulation that manual trial planning was generally not useful for creating vectors for arrival metering. Metering situations are very tactical and controllers do not have time to operate the Trial Planner."

Analyzing this trial planning approach further reveals that it does not remove any procedural steps from the current day procedure. The graphical plan construction augments step (2) in the current day procedure, which is to determine the appropriate heading for the delay vector. In the current day environment this heading determination only takes very little time, probably less than a second, because controllers are very familiar with their airspace and their typical delay routings. In the current day environment controllers can issue the heading immediately, whereas the trial planner adds an additional step by asking the controller to construct the route and entering it into the ground system.

The other step augmented by the trial planning process is step (5): determining the turn back point. With the trial planner the controller can pre-determine the turn back point and instruct the aircraft to proceed to the metering fix, when it approaches the point. However, even with a trial planner the controller still has to make sure not to miss the pre-planned turn point.

The fact that this trial planning implementation basically added work and did not remove any procedural steps, explains to some extend why it was not considered useful for delay vectoring - a high workload situation. Another important factor in this early implementation was the tool responsiveness. Every time the controller drags the turn back point to a new position a new trajectory is computed and conflict probed. This computation often took several seconds, making the tool appear rather slow.

In order to make ground based automation actually helpful in delay vectoring two areas could be targeted for improvement: Speeding up the route determination process and eliminating the need for the controller to call the aircraft's turn back.

\subsection{Ground-based automatic advisories}

One approach at addressing these problems has been pursued in the development of the CTAS Enroute Descent Advisor (EDA) [9] [10]. In this approach the controller enters the initial heading, but the automation tries to 
compute a turn back point automatically that will provide a conflict free flight path and meet the desired STA. The controller communicates the initial heading and the turn back point to the flight crew. One approach is to use the time at which the turn should occur. In this case the pilot initiates the turn back at the given time.

While this approach addresses some of the problems described above it may create some new problems: The ground automation creates a conflict free flight path based on the current traffic situation. However, controllers often accept creating medium range conflicts (e.g. $>4$ minutes) when they know that the flight path of the conflict aircraft will have to be changed soon. When for example several aircraft have to be delayed, controllers turn the first aircraft, then the second, then the third. The first aircraft's turn would most likely create a medium-range conflict if the other aircraft would not be turned later. If the ground automation does not account for these kinds of strategies the controller may not understand the provided solution and lose faith in the automation.

The second important factor to be considered in the EDA development is how the turn back point will be communicated in a voice environment. If the description of the turn back location is not compatible with the on-board automation (FMS), it cannot be preprogrammed and the on-board automation cannot be used. For example the communication of the turn back time would require pilots to monitor the flight progress to initiate the turn back at the appropriate time. If the pilots miss the turn back point the trajectory is no longer flown as planned and the controllers may have to do another adjustment, causing additional workload. Therefore, in order to use voice communication in this procedure it would be useful if the turn back point was specified in a FMS compatible way, e.g. using or referencing waypoints retrieved from the data base. In this case the flight crew could use their on-board automation throughout the procedure.

\subsection{Ground-based trial planning with integrated data link}

Another approach that requires advanced technology is to communicate the new flight path via data link to the aircraft. This procedure was used in simulations for a number of years during CTAS/FMS integration and Distributed Air/Ground Traffic Management (DAG-TM) research. Since no ground automation was sophisticated enough to generate the flight paths automatically, manual trial planning was used.

The procedure involves the following steps

(1) The air traffic controller detects that the aircraft will arrive earlier than it should using the metering delay information provided on the display.
(2) The air traffic controller determines an appropriate trajectory that absorbs the delay using a graphical trial planning tool

(3) The controller sends the trajectory via data link to the flight deck and the ground automation

(4) The flight crew loads the trajectory into the FMS, reviews it and -if acceptable- accepts and executes it. The airborne automation flies the trajectory and communicates it back to the ground automation

The data link integration has a number of desirable properties. Three steps have been eliminated compared to the current day procedure. Since the complete flight path is communicated the controllers and the pilots do not have to monitor as closely to make sure the turn back point is not missed. Both, the ground automation and the airborne automation are informed about the aircraft's flight path and provide additional support in terms of vertical path planning and conformance monitoring.

\subsubsection{Evaluation of initial prototype (1999-2003)}

An initial implementation of metering with the data link integrated trial planning tool was used in different simulations between 1999 and 2003. From a flight deck perspective the procedure was desirable and acceptable [11]. From a ground side perspective controllers could see the potential, but were unsatisfied with the responsiveness of the tool [12]. Table 1 shows the usability and usefulness results from post simulation questionnaires.

Table 1: Route modification tool

Usability: Very easy to use (5) Very difficult to use (1)" Usefulness: Vital(5) Unnecessary (1)

\begin{tabular}{|c|c|c|c|c|c|}
\hline $\begin{array}{c}\text { Route Modification } \\
\text { Tool }\end{array}$ & Mean & $\begin{array}{c}\text { Std. } \\
\text { Err. }\end{array}$ & Med. & $\begin{array}{c}\text { Std. } \\
\text { Dev }\end{array}$ & Count \\
\hline Usability & 3.5 & 0.45 & 4 & 1.61 & 13 \\
\hline Usefulness & 3.7 & 0.40 & 4 & 1.44 & 13 \\
\hline
\end{tabular}

Thirteen controllers gave the tool an average usability rating of 3.5 and an average usefulness rating of 3.7 , making it somewhat usable and useful. These ratings are reflected in typical controller comments.

Controller 1: "Single most workload reducing part of tool appears to be datalink. Trial plans (when working) for speed and routes were a nice tool for TBM". (TBM = time based metering)

Controller 2: "Route MOD is too slow. I cannot emphasize this enough. This is a phenomenal tool but responds far too slowly at critical junctures". 
Controller 3: "As with any automation, workload increases due to database maintenance. Workload somewhat decreases due to calculations produce one-time clearance. This eliminates need for vast experience at control position"

While the data link integration shows some good potential the slow responsiveness of the tool still remained a major problem. This was addressed with a complete redesign of the software architecture of the tool.

\subsubsection{Evaluation of final integrated prototype (2004)}

The trial planner was re-implemented using a different architecture, platform and trajectory generation algorithm. This prototype is fully integrated with an emulation of a state of the art controller display and provides quasi immediate feedback during the trial planning process. When a controller drags a waypoint along the route the new trajectory and conflict feedback is typically provided within less than $100 \mathrm{~ms}$. This revised tool was part of DAG-TM simulations in 2004 and received the maximum rating of 5 (very usable, very useful) from all four controllers that used it.

The data link integrated toolset for the controllers used in this simulation contained altitude trial planning, speed advisories and data linked frequency changes in addition to the route trial planning function. The complete set of technologies is described in [13]. Three of the four controller noted specific comments on this type of integrated toolset:

Controller A: "implement this today would save time, money and make controllers' job safer and better and give more time for conflict resolution and custom service."

Controller B: "CPDLC's reduction of frequency congestion was a very useful workload reduction tool that allowed one the time that was necessary to use the other tools." (CPDLC $=$ Controller Pilot Data Link Communication)

Controller D: "Uplinking route and altitude changes reduced controller workload greatly"

Clearly, the prototype was very well accepted. While controller acceptance is important, objective improvements need to be achieved to justify investing in the automation. These objective improvements were demonstrated as aircraft vectoring was practically eliminated and controllers could handle traffic loads of approximately $150 \%$ of today's values without excessive workload [13].

\section{Where do we go from here?}

The preceding section has illustrated how an envisioned tool for arrival metering that was initially considered "generally not useful for delay vectoring" was developed through human-automation integration analysis, simulations and prototypes such that it received the highest usability and useful ratings possible. Two requirements were identified: The tool must be highly responsive and well integrated with the ground-based and airborne automation.

This example also indicates that a partial or immature implementation of this kind of automation may likely have an adverse effect. The automation may be considered useless or unusable and never be evaluated to its full potential. In order to avoid these kinds of problems we propose a multi-stage research process centered on rapid prototyping and simulation methods as described in the next section.

\section{Research process}

The research process focuses on the operational aspects of new concepts, the interactions of humans and humans, humans and automation, and automation and automation in a highly complex environment. It is aimed at evaluating feasibility, validating modeling assumptions, and specifying requirements on automation and procedures to enable the desired operations. The process enables rapid evaluation of envisioned air traffic concepts utilizing expert knowledge at critical stages. The main phases of the research process are depicted in Figure 2.

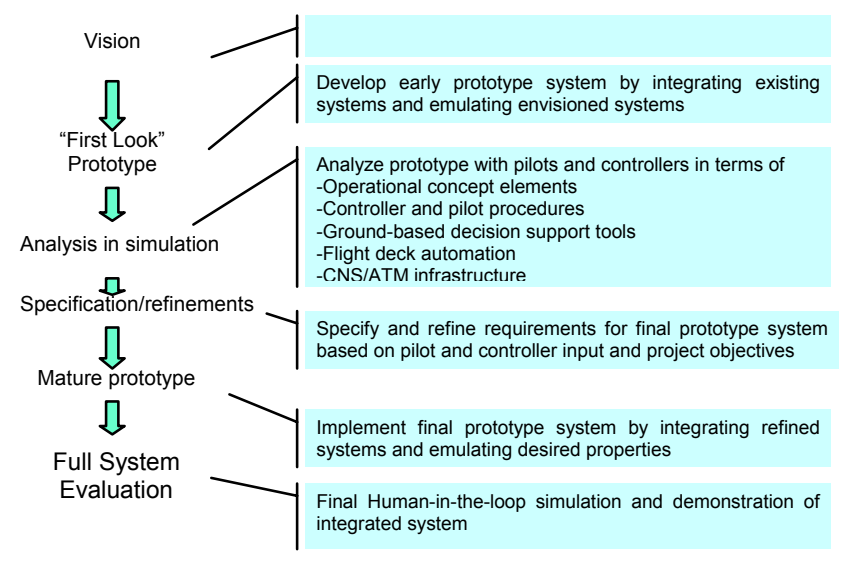

Figure 2. Research Process

The vision is typically formulated by a number of experts - based on experience and prior research. The vision is laid out in a concept definition describing the target system at a high level.

This envisioned system is then turned into an early prototype by integrating simplified mock-ups of the primary new technologies into an emulation of the existing environment. This "first look" prototype provides an early reality check of concept feasibility and allows practitioners (i.e. pilots and controllers) to interact with the system in a 
"quasi" operational environment. It also provides an opportunity to demonstrate the system to all potential stakeholders. An early simulation analysis represents a possible decision point for either proceeding with the concept exploration as planned, redirecting its focus, or terminating this particular research at an early stage.

A simulation with pilots and controllers and other relevant parties such as airline dispatchers or air traffic managers is used to refine the concept and specify requirements for the mature system. The identified requirements are then turned into a revised prototype that integrates all relevant properties for evaluating the concept in a simulated operational target environment. This prototype is designed towards providing the desired look and feel to the operators and addressing all procedural issues. It is not intended to be an operational implementation of the automation. Thus, the software does not have to meet the high requirements imposed on operational systems and can be implemented in a straightforward and efficient manner.

The final human-in-the-loop evaluation is designed to measure whether the envisioned system meets the objectives targeted in the original concept definition. It also identifies the final specification for the required system components as well as subsystems that might be implemented in the near-term and could provide immediate benefits.

\section{Concluding Remarks}

This paper has scratched the surface of the many aspects related to the design of integrated air/ground automation. There is a tremendous push for integrating new automation into the Next Generation Air Transportation System. Introducing effective automation into this complex and multi-layered environment, however, requires a thorough evaluation of the new roles, procedures and technologies. A human-automation integration centered research process using expert analysis, rapid prototyping, and simulation can provide invaluable insights to make this technology driven air traffic revolution successful.

\section{References}

[1] Joint Planning and Development Office 2004, Next Generation Air Transportation System Integrated Plan. http://www.jpdo.aero

[2] European Commission 2005 "The Single European Sky Implementation Programme: SESAME" http://europa.eu.int/comm/transport/air/single_sky/sesame/ doc/sesame_web_light.pdf and Eurocontrol 2005 http://www.eurocontrol.int/eec/public/standard_page/resear ch_areas.html
[3] NASA (1999) Concept Definition for Distributed Air/Ground Traffic Management (DAG-TM), http://www.asc.nasa.gov/aatt/dagconop.pdf

[4] FAA/Eurocontrol (2001) Action Plan 1 FAA/Eurocontrol Cooperative R\&D, Principles of Operation for the Use of Airborne Separation Assurance Systems Version 7.1, 19 June 2001

[5] Erzberger, H. (2004) Transforming the NAS: The next generation air traffic control system. Air Traffic Control Quarterly, Vol. 10(4) 355-378. Arlington, VA

[6] Billings, C.E. (1991), "Human-centered aircraft automation: A concept and guidelines," NASA Technical Memorandum 103885, Moffett Field, CA: NASA- Ames Research Center.

[7] Erzberger, H., T.J. Davis, and S.M. Green (1993) Design of Center-TRACON Automation System, AGARD Meeting on Machine Intelligence in ATM, Berlin, Germany, May, and http://ctas.arc.nasa.gov

[8] McNally, B. D., H. Erzberger, R. E. Bach, and W. Chan, A Controller Tool for Transition Airspace, AIAA Guidance, Navigation, and Control Conference, Portland, OR, August 9-11, 1999 (AIAA-99-4298).

[9] Green, S. M., and R. A. Vivona, En route Descent Advisor Concept for Arrival Metering, AIAA 2001-4114, Guidance, Navigation, and Control Conference, Montreal, Canada, August 2001.

[10] Coppenbarger R., Lanier R., Sweet D. and S. Dorsky Design and Development of the En Route Descent Advisor (EDA) for Conflict-Free Arrival Metering AIAA-2004-4875 AIAA GNC Conference, Providence, Rhode Island, Aug. 16-19, 2004

[11] Crane, B, T. Prevot and E. Palmer. (1999) Flight Crew Factors for CTAS/FMS Integration in the Terminal Airspace. NASA Technical Memorandum 2000-209607, NASA Ames Research Center, Moffett Field, CA.

[12] Prevot T., P. Lee, T. Callantine, N. Smith, and E. Palmer (2003) Trajectory-Oriented Time-Based Arrival Operations: Results and Recommendations, ATM2003, FAA/Eurocontrol R\&D Seminar, Budapest, Hungary

[13] Prevot T., Lee P, Smith, N. and Palmer E. (2005), ATC Technologies for Controller-Managed and Autonomous Flight Operations. AIAA Guidance Naviation and Control Conference, San Francisco, CA 2005 (in preparation) 